EPJ Web of Conferences 66, 02044 (2014)

DOI: $10.1051 /$ epjconf/20146602044

(C) Owned by the authors, published by EDP Sciences, 2014

\title{
Scissors strength in the quasi-continuum of actinides
}

\author{
M. Guttormsen ${ }^{1, a}$, L.A. Bernstein ${ }^{2}$, A. Bürger ${ }^{1}$, A. Görgen ${ }^{1}$, F. Gunsing ${ }^{3}$, T.W. Hagen ${ }^{1}$, \\ A.C. Larsen ${ }^{1}$, T. Renstrøm ${ }^{1}$, S. Siem ${ }^{1}$, M. Wiedeking ${ }^{4}$, and J.N. Wilson ${ }^{5}$ \\ ${ }^{1}$ Department of Physics, University of Oslo, N-0316 Oslo, Norway \\ ${ }^{2}$ Lawrence Livermore National Laboratory, 7000 East Avenue, Livermore, CA 94550-9234, USA \\ ${ }^{3}$ CEA Saclay, DSM/Irfu/SPhN, F-91191 Gif-sur-Yvette Cedex, France \\ ${ }^{4}$ iThemba LABS, P.O. Box 722, 7129 Somerset West, South Africa \\ ${ }^{5}$ Institut de Physique Nucleaire d'Orsay, Bat. 100, 15 rue G. Glemenceau, 91406 Orsay Cedex, France
}

\begin{abstract}
The M1-scissors resonance has been measured for the first time in the quasicontinuum of actinides. The strength and position of the resonances in ${ }^{231,232,233} \mathrm{Th}$ were determined by particle- $\gamma$ coincidences using deuteron induced reactions on $\mathrm{a}^{232} \mathrm{Th}$ target. The residual nuclei show a strong integrated strength of $B_{M 1}=9-11 \mu_{n}^{2}$ in the $E_{\gamma}=$ $1.0-3.5 \mathrm{MeV}$ region. The presence of the scissors resonance modifies significantly the $(\mathrm{n}, \gamma)$ cross section, which has impact on fuel-cycle simulations of fast nuclear reactors and nucleosynthesis in explosive stellar environments.
\end{abstract}

\section{Introduction}

The $\gamma$ decay of excited atomic nuclei is to a large extend governed by collective transitions. In particular, the enhanced E2 $\gamma$ decay of rotational bands is well known. The softest collective M1 mode, the scissors resonance (SR), appears when the deformed proton and neutron clouds oscillate against each other like the blades of a scissors. Such an isovector collective motion was first predicted by Lo Iudice and Palumbo [1].

The $\gamma$-ray strength function $(\gamma \mathrm{SF})$ is a measure of the average electromagnetic properties of $\gamma$ transitions in the quasi-continuum. Another important concept in the study of quasi-continuum is the level density (LD) as function of excitation energy. These two functions are crucial inputs for calculating neutron-induced reaction cross sections for neutron energies starting from the keV range. It is particularly important to extrapolate in cases where measured data are insufficient or lacking. Such types of extrapolation is relevant for future and existing nuclear power reactors [2], and in stellar nucleosynthesis $[3,4]$.

The spins and parities of some SR states of actinides have been measured with reactions on the ground state. Several SR states in ${ }^{232} \mathrm{Th}$ and ${ }^{235,236,238} \mathrm{U}$ have been determined in $\left(\gamma, \gamma^{\prime}\right)$ and $\left(e, e^{\prime}\right)$ reactions [5-7] with typical strengths of $B_{M 1} \sim 3 \mu_{N}^{2}$. Since these experiments rest on the identification of single states in an energy region of $10^{4}-10^{5}$ levels per $\mathrm{MeV}$, one could expect that not all the strength has been resolved as $\gamma$ or electron peaks. In addition, we expect lower strength for SR built on the ground state compared to SR transitions in the quasi-continuum. Recently, a review of $\left(\gamma, \gamma^{\prime}\right)$ and $\left(e, e^{\prime}\right)$ experiments and various models has been given [8].

\footnotetext{
ae-mail: magne.guttormsen@fys.uio.no
} 


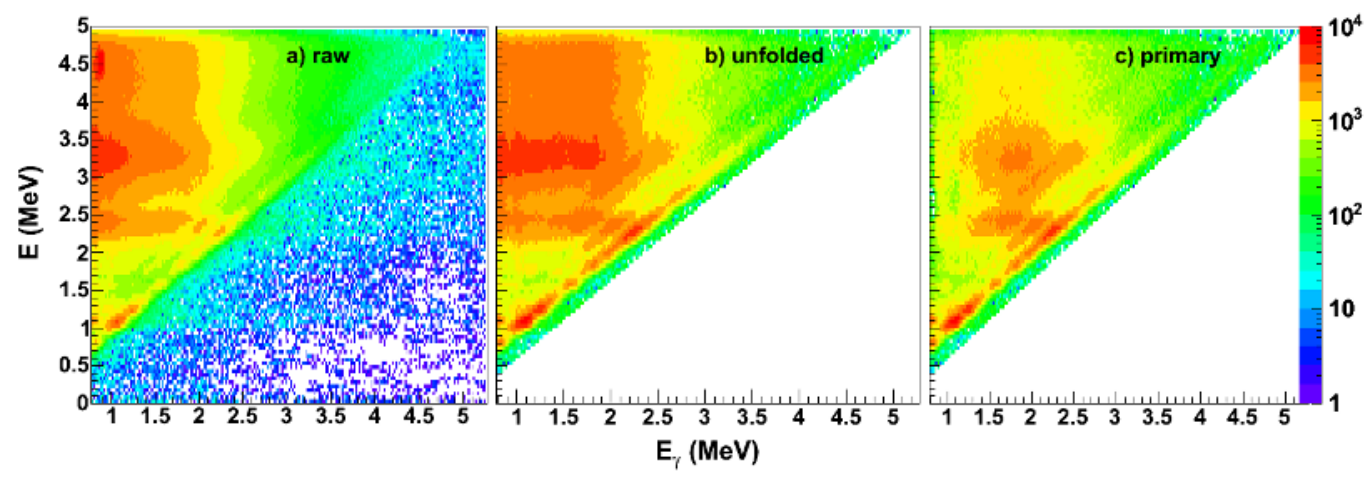

Figure 1. Initial excitation energy $(E)$ versus $\gamma$-ray energy $\left(E_{\gamma}\right)$ from particle- $\gamma$ coincidences recorded with the ${ }^{232} \mathrm{Th}(\mathrm{d}, \mathrm{p} \gamma)^{233} \mathrm{Th}$ reaction. The raw $\gamma$-ray spectra (a) are first unfolded (b) by the NaI response function. In the last step (c), the primary or first-generation $\gamma$-ray spectra are extracted as function of excitation energy.

\section{Experimental method}

The experiments were conducted at the Oslo Cyclotron Laboratory (OCL) with a 12-MeV deuteron and a $24-\mathrm{MeV}^{3} \mathrm{He}$ beam bombarding a self-supporting target of ${ }^{232} \mathrm{Th}$ with thickness of 0.968 $\mathrm{mg} / \mathrm{cm}^{2}$. Particle- $\gamma$ coincidences were measured with the SiRi particle telescope and the CACTUS $\gamma$ detector systems $[9,10]$. The $64 \mathrm{SiRi}$ particle telescope detectors were placed in backward direction, covering eight angles from $\theta=126$ to $140^{\circ}$ relative to the beam axis. The CACTUS array consists of 28 collimated 5" $\times 5 " \mathrm{NaI}(\mathrm{Tl})$ detectors with a total efficiency of $15.2 \%$ at $E_{\gamma}=1.33 \mathrm{MeV}$.

Figure 1 demonstrates the first steps of the Oslo Method applied to ${ }^{233} \mathrm{Th}$. For reactions with one charged ejectile, the excitation energy $E$ is easily calculated from the kinematics. In the event-sorting routine, we increment the $\gamma$-spectra for each excitation bin $E$, which in turn are unfolded using the NaI-response function. An iterative subtraction technique [11] has been developed to separate out the first-generation (primary) $\gamma$ transitions from the total $\gamma$ cascade. It is assumed that the $\gamma$-decay pattern is the same whether the levels were initiated directly by the nuclear reaction or by the decay from higher-lying states. In the last step, the first-generation matrix $P\left(E, E_{\gamma}\right)$ is disentangled into level density and $\gamma$-ray strength function $[12,13]$.

In the quasi-continuum, we assume that $P$ is proportional to the level density at the final excitation energy $\rho\left(E-E_{\gamma}\right)$ in accordance with Fermi's golden rule. Furthermore, the decay is governed by the $\gamma$-transmission coefficient $\mathcal{T}\left(E \rightarrow E-E_{\gamma}\right)$, which according to the Brink hypothesis, is independent of excitation energy. The $\gamma \mathrm{SF}$ is connected to the transmission coefficient by $f\left(E_{\gamma}\right)=\mathcal{T}\left(E_{\gamma}\right) / 2 \pi E_{\gamma}^{3}$. Thus, the decay probability is given by

$$
P\left(E, E_{\gamma}\right) \propto E_{\gamma}^{3} f\left(E_{\gamma}\right) \rho\left(E-E_{\gamma}\right)
$$

This allows a simultaneous least $\chi^{2}$-fit of the two one-dimensional functions $E_{\gamma}^{3} f\left(E_{\gamma}\right)$ and $\rho(E)$ to the two-dimensional matrix $P$.

The level density of thorium and uranium isotopes is recently found to be exponentially increasing as function of excitation energy [14]. A closer examination of these level densities indicates that a 
continuous breaking of Cooper pairs is responsible for the constant temperature. However, in this work we will only focus on the scissors component of the $\gamma \mathrm{SF}$.

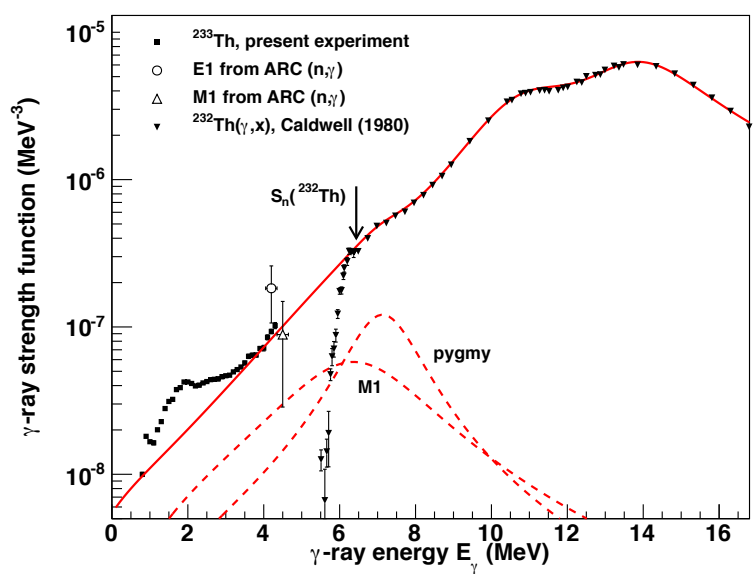

Figure 2. Normalization of the $\gamma$-ray strength functions of ${ }^{233} \mathrm{Th}$. For comparison, the $(\gamma$, abs) reaction on ${ }^{232} \mathrm{Th}$ [16] are shown (triangles). The solid red line is an estimate of the tail of the ${ }^{232} \mathrm{Th}$ GEDR (including the GMDR and a pygmy-like resonance) used for the "background" of the SR. Also two data points (open symbols) from the $(n, \gamma)$ reactions are shown [17].

\section{Scissors resonance}

The experimental $\gamma$ SFs have been published previously [15], however, several improvements are performed in the present work; $(i)$ in the unfolding procedure, new and better response functions deduced from reactions on ${ }^{56,57} \mathrm{Fe},{ }^{28} \mathrm{Si},{ }^{16} \mathrm{O}$ and ${ }^{12} \mathrm{C}$ have been applied, and (ii) newer photonuclear reactions [16] are taken into account in order to estimate the E1 component from the GEDR in the $\gamma \mathrm{SF}$.

The $\gamma \mathrm{SF}$ has to be normalized, since only its functional form is extracted in the Oslo method. Usually, the average capture neutron spacings $D_{0}$ and the average $\gamma$ width $\left\langle\Gamma_{\gamma}\left(S_{n}\right)\right\rangle$ are utilized in the normalization procedure $[12,13]$. However, since both the reaction and the total nuclear spin distribution is rather unclear in these heavy nuclei, we have chosen to normalize directly to the expected "background", shown as a red curve in Fig. 2. The "background" represents the sum of components that one expects to be present as a "background" to the SR $\gamma \mathrm{SF}$. Since this contribution is varying very little between neighboring isotopes, we use the photonuclear data of ${ }^{232} \mathrm{Th}$ for all ${ }^{231-233} \mathrm{Th}$ isotopes.

Figure 3 shows the experimental $\gamma \mathrm{SF}$ for ${ }^{231-233} \mathrm{Th}$. The three isotopes show qualitatively the same behavior. The resonances cover the energy region of $E_{\gamma} \approx 1.0-3.5 \mathrm{MeV}$ with an energy centroid of about $E_{\gamma} \approx 2.2 \mathrm{MeV}$. The SR strength is typically $B_{M 1}=9-11 \mu_{n}^{2}$, which is $3-4 \mu_{n}^{2}$ lower than previously published [15].

The scissors resonance is split into two Lorentzians separated by $\Delta E_{\gamma} \approx 0.85 \mathrm{MeV}$. The ratio of strengths between the lower and higher components is about 1.8. To our knowledge, there exist no fundamental and quantitative description of the observed splitting. 


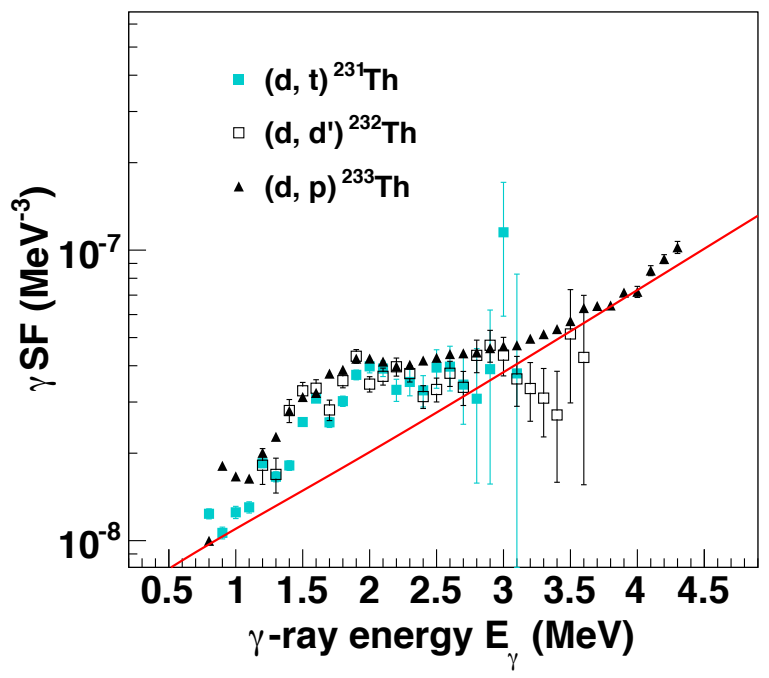

Figure 3. Gamma-ray strength functions for ${ }^{231-233} \mathrm{Th}$.

The large $\gamma$-ray strength of the SR enhances the $\gamma$-decay branch above the neutron separation energy. In particular, preliminary simulations with the TALYS code indicate an increase in the (n, $\gamma$ ) cross section of about 100-200 mb. A more comprehensive work on the $\gamma \mathrm{SFs}$ in the actinides is under preparation.

\section{References}

[1] N. Lo Iudice and F. Palumbo, Phys. Rev. Lett. 41, 1532 (1978).

[2] M.B. Chadwick et al., Nucl. Data Sheets 112, 2887 (2011).

[3] M. Arnould, S. Goriely and K. Takahashi, Phys. Rep. 450, 97 (2007).

[4] F. Käppeler et al., Rev. Mod. Phys. 83, 157 (2011).

[5] R.D. Heil et al., Nucl. Phys. A 476, 39 (1988).

[6] J. Margraf et al., Phys. Rev. C 42, 771 (1990).

[7] O. Yevetska et al., Phys. Rev. C 81, 044309 (2010).

[8] K. Heyde et al., Rev. Mod. Phys. 82, 2365 (2010).

[9] M. Guttormsen et al., Nucl. Instrum. Methods Phys. Res. A 648, 168 (2011).

[10] M. Guttormsen et al., Phys. Scr. T 32, 54 (1990).

[11] M. Guttormsen et al., Nucl. Instrum. Methods Phys. Res. A 255, 518 (1987).

[12] A. Schiller et al., Instrum. Methods Phys. Res. A 447, 498 (2000).

[13] A.C. Larsen et al., Phys. Rev. C 83, 034315 (2011).

[14] M. Guttormsen et al., Phys. Rev. C 88, 024307 (2013).

[15] M. Guttormsen et al., Phys. Rev. Lett. 109, 162503 (2012).

[16] J.T. Caldwell et al., Phys. Rev. C 21, 1215 (1980).

[17] R. Capote et al., Reference Input Parameter Library, RIPL-3 (2012). 